\title{
A Barycenter Control Method for the Bioinspired Forest Chassis Robot on Slope
}

\author{
Tingting Sui $\mathbb{D}^{1,2}$ Jinhao Liu $\mathbb{D}^{1,2}$ Jianli Wang $\mathbb{D}^{3}$, and Jianting Zhang $\mathbb{D}^{1,2}$ \\ ${ }^{1}$ School of Technology, Beijing Forestry University, Beijing 100083, China \\ ${ }^{2}$ Critical Lab of State Forestry Administration on Forestry Equipment and Automation, Beijing 100083, China \\ ${ }^{3}$ School of Materials Science and Mechanical Engineering, Beijing Technology and Business University, Beijing 100083, China
}

Correspondence should be addressed to Jinhao Liu; liujinhao@bjfu.edu.cn and Jianli Wang; wangjianli@btbu.edu.cn

Received 16 February 2021; Accepted 5 April 2021; Published 30 April 2021

Academic Editor: Oscar Reinoso

Copyright ( $\odot 2021$ Tingting Sui et al. This is an open access article distributed under the Creative Commons Attribution License, which permits unrestricted use, distribution, and reproduction in any medium, provided the original work is properly cited.

To improve the stability of forestry chassis on the slope, a chassis-installed barycenter adjustable mechanism (BAM) is designed, and the control method of the counterweight is proposed to make the chassis barycenter move suitably to achieve the design purpose. The kinematic analysis of BAM is carried out, and the relationship between the translation, rotation, and vertical displacement of counterweight and the chassis barycenter is calculated. Furthermore, the variation curves obtained in Matlab show the barycenter can translate $100 \mathrm{~mm}$, rotate from 0 to 360 degrees, and lower about $180 \mathrm{~mm}$ in the vertical direction. Adams is adopted to complete the kinematics simulation of the chassis, indicating that the control method can effectively adjust the barycenter position. Finally, experiments are carried out under slope conditions to analyze chassis stability by testing plantar pressure. The results show that forest chassis using the barycenter control method helps keep stable on the slope of 15 degrees, much better than standard normal chassis.

\section{Introduction}

The site conditions of forestry production are characterized by rugged terrain and slope conditions in China. Stable movement of equipment chassis is an essential guarantee for the regular operation of forestry machinery. However, research on unique vehicle chassis in forestry terrain is relatively weak compared with that at home. Due to the significant terrain differences, the forest vehicle chassis developed by other countries has insufficient adaptability in China's forests $[1,2]$. Therefore, it is imperative to develop unique vehicle chassis with better operation stability for sloping forest land [3].

Traditional wheeled chassis have severe mobility restrictions on rough terrain [4]. The articulated mining vehicle researched by Zhou et al. can keep the front and rear tracks in good contact with the ground to ensure that it is suitable for complex terrain [5]. The six-wheel swing arm forestry chassis proposed by Sun Zhibo can improve the chassis stability by changing its posture and ensure that it still has the good adaptive ability on rough terrain [6]. Gao et al. designed the obstacle crossing chassis (V2-HVPC) with balanced rocker arm suspension. The chassis adjusted the wheel base according to the road conditions to improve the terrain trafficability. Also, the chassis' driving stability on uneven road surfaces was studied and verified $[7,8]$.

Compared with wheeled or tracked chassis, quadruped chassis has outstanding advantages in terrain adaptability and environmental protection. At the same time, it is difficult for quadruped chassis to keep stable walking on the slope. The main problem is the barycenter is high, so that the chassis is easy to overturn. Because of quadruped chassis stability on the slope, many methods are proposed to calculate the stability, including the stability margin (SM), the zero moment point (ZMP) theory, and Kalakrishnan's walking gait of the quadruped chassis by planning the ZMP trajectory [9-12]. The position and height of barycenter are important factors affecting the chassis stability, so it is an excellent idea to consider how to use centroid adjustment to improve the operation stability of quadruped chassis under 
slope conditions. In essence, suitable structure design is the basis of motion stability for chassis.

It is a standard method to add a barycenter adjustment mechanism to assist the control of the whole machine's barycenter, such as the counterweight used in the construction site and the inertial flywheel to adjust the attitude of the satellite. In addition, the bionic engineering method is widely adopted to develop the barycenter adjustment mechanism by inspiration from nature. As far as quadrupeds are concerned, all kinds of quadrupeds can adjust the barycenter, not only by the forward and backward movement of limbs and trunk but also by other structures and organs. When the cheetah runs, its tail helps to maintain stability. Obviously, in the process of sharp turning, the cheetah will quickly swing its tail to the other side to prevent instability. The Cheetah with mounted 2-DOF tail developed by MIT is used to study the tail and body interaction. The research results show that tail swing can reduce the motion system's lateral disturbance [13]. Like cheetahs, when a cat falls or jumps from a height, it can also adjust its body posture by swinging its tail quickly to ensure its balance [14]. In all kinds of accidental falls, lizards can adjust their tails to make their bodies nearly level to ensure that they can land on the ground with their feet. A UC Berkeley research team proposed a car with a tail by studying the effect of lizards' and dinosaurs' tails on adjusting body balance. Comparative experiments show that the car with a tail has better spatial stability [15-17]. According to the kangaroo's physiological structure, movement characteristics, and the tail's role in the movement, the researchers proposed a bionic jumping robot model with a tail. The analysis results show that the tail can adjust the jumping posture and degree and reduce the touchdown force. The research of Bionic Kangaroo has proved that the tail can significantly change the robot's attitude in the air [18]. There are many other studies on improving system stability by using adjustment mechanisms $[19,20]$. Goats can adjust the barycenter position using the head, neck, and other physiological structures. The goats live in the rugged mountain environment, and the rock antelope is a modern relative of a goat. However, the study found that the goat's muscle is more developed than that of the rock antelope, especially the shoulder and neck. Also, the short and robust limbs and prominent shoulder muscles are the goat's apparent characteristics [21]. Compared with other climbing animals, such as bighorn sheep, the goat has a lower barycenter [22]. Anatomically, a goat's chest height is much lower than that of bighorn sheep, and the hoof area of a goat is more prominent [23]. Combined with many factors, including a large proportion of forelimbs, slightly lower forebody, long neck, and developed shoulder muscle tissue, it is helpful for goats to climb or pass through complex terrain. Ryan $\mathrm{T}$ analyzed the climbing mechanism of goats. In the stage of forelimb pedaling and extension, the trunk barycenter should be as close to the ground as possible.

During the pull-up phase, the trunk barycenter experienced vertical and horizontal movements of about $23 \mathrm{~cm}$ and $15 \mathrm{~cm}$, respectively. When the hind limbs are pushed off the ground, the vertical distance of the trunk barycenter's vertical distance relative to the hind hooves is about $48 \mathrm{~cm}$, and the horizontal distance is about $38 \mathrm{~cm}$ [24]. The research shows that goats can keep their body stable by adjusting the barycenter all the time when passing through complex terrain. Zhang Fu et al. analyzed the movement characteristics of goats on the slope. The results show that the walking gait can be optimized by adjusting the barycenter position. In addition, the influence of goat head control on the machine operation is studied, and the control strategy to improve the balance performance is obtained $[25,26]$.

Inspired by the barycenter adjustment of goats passing complex terrain, this paper proposes a bionic chassis installed barycenter adjustable mechanism (BAM) based on the auxiliary effect of goat head and neck on barycenter adjustment. Based on the stability margin, a new method to adjust the chassis barycenter is proposed, realized by changing the BAM's parameters. The advantage of this method is that the control is simple. Two aspects of barycenter adjustment can be realized together: on the one hand, the chassis barycenter can be adjusted to make the projection point fall inside the support polygon on the ground to improve the stability margin; on the other hand, it can lower the centroid height to prevent the chassis from rolling over. The correctness and effectiveness of the proposed method are verified by simulation. Based on the model drawings and simulation results, the prototype is manufactured and experiments are conducted. The results prove the validity of the structure and method.

\section{Materials and Methods}

2.1. Structure Design. The stability of mechanical operation is poor on slope terrain. The barycenter of the chassis directly affects its stability. Based on this, the design requirement that the chassis system has better stability on the slope is put forward. The forestry chassis with BAM is mainly composed of legs, frame, and drive system, as shown in Figure 1.

The barycenter's projection on a slope does not coincide with that on flat ground when the chassis is stationary. If the chassis starts to move, the barycenter shifts that are prone to overturn. The BAM needs to help keep the chassis barycenter within the stability margin by adjusting the counterweight position to avoid the chassis rolling over. Based on this, a new 3-DOF barycenter balance mechanism that can realize the translation $(R)$, rotation $\left(\theta_{a}\right)$, and vertical $(\sigma)$ motion of the counterweight is proposed, as shown in Figure 2. The counterweight is driven by motors to change the chassis barycenter position as needed by adjusting $R, \theta_{a}$, and $\sigma$.

\subsection{Motion Analysis of the Barycenter Adjustable Mechanism.} To analyze the barycenter position of BAM, its coordinate system should be established first. It is noted that BAM is considered a symmetrical structure. The influence of motor is ignored due to its small mass. Based on motion features, parts are classified into three groups: connecting rods, connecting plate, and slide rail; counterweight; and motor 3. Therefore, $\{G\}$ of BAM's coordinate system is defined 


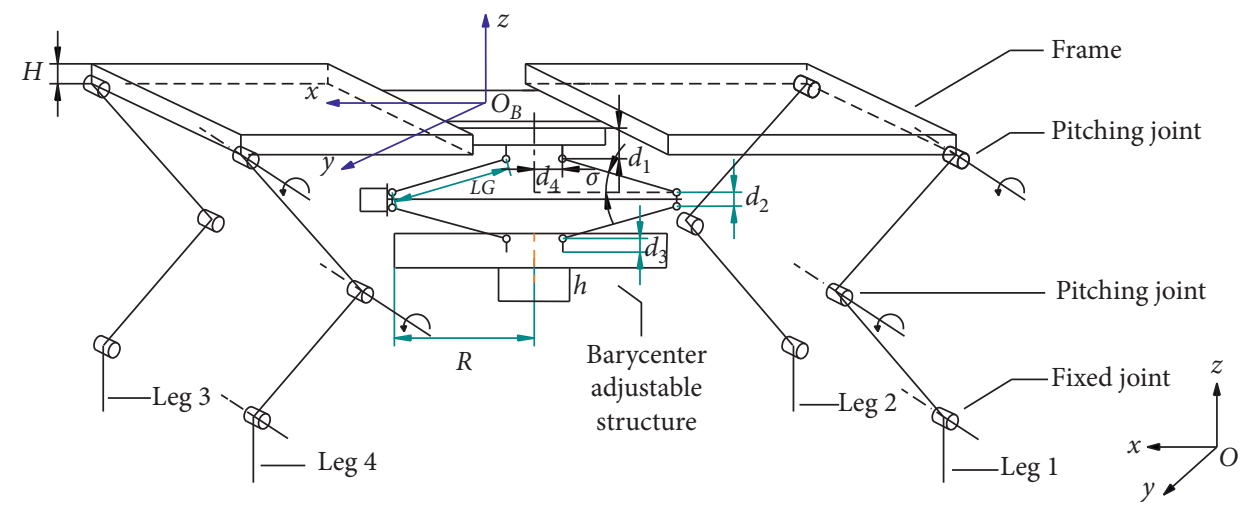

FIGURE 1: Sketch of forestry chassis with BAM.

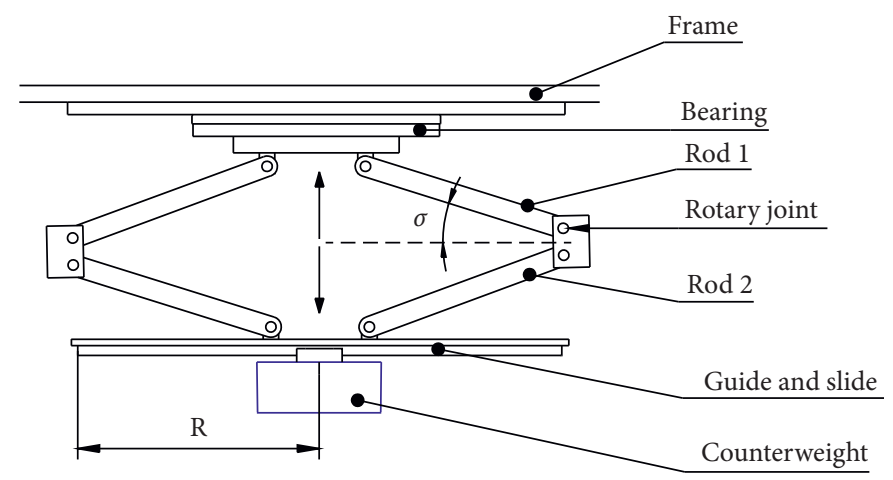

(a)

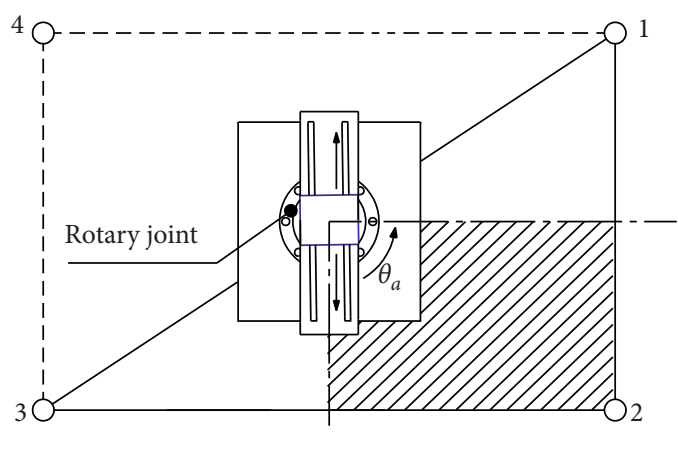

(b)

FIGURE 2: Model of barycenter balance structure.

TABLE 1: Parameter of the barycenter balance mechanism.

\begin{tabular}{lcc}
\hline Structure & Para & Unit \\
\hline Total mass of BAM & $M_{G}$ & $\mathrm{~kg}$ \\
Mass of 8 links & $M_{\mathrm{a}}$ & $\mathrm{kg}$ \\
Mass of connecting plate & $M_{\mathrm{b}}$ & $\mathrm{kg}$ \\
Mass of counterweight & $M_{\mathrm{c}}$ & $\mathrm{kg}$ \\
Rotate angle of motor 1 & $\theta_{a}$ & $\left(^{\circ}\right)$ \\
Angle between link and level & $\sigma$ & $\left(_{0}\right.$ \\
Mass of frame & $\mathrm{M}_{\mathrm{G}}$ & $\mathrm{kg}$ \\
Length of link & $\mathrm{mm}$ \\
Height of counterweight & $\mathrm{mm}$ \\
Range of counterweight & $R$ & $\mathrm{~mm}$ \\
Distance between plate and frame & $h_{\mathrm{G}}$ & $\mathrm{mm}$ \\
Height of frame & $\mathrm{m}$ & $\mathrm{mm}$ \\
\hline
\end{tabular}

with its origin at the geometric center of the connecting plate. The simplified model and coordinate system are shown in Figure 1, and parameters are presented in Table 1.

According to the working principle of BAM, the distance between the connecting plate and the chassis frame can be obtained:

$$
h_{G}=2 L_{G} \times \sin \sigma+d_{2}+d_{1} .
$$

Let the barycentric coordinate of BAM is $G_{B}\left(x_{B}, y_{B}, z_{B}\right)$, and that of $M_{a}$ is $G_{a}\left(x_{a}, y_{a}, z_{a}\right), M_{b}$ is $G_{b}\left(x_{b}, y_{b}, z_{b}\right)$, and $M_{c}$ is $G_{c}\left(x_{c}, y_{c}, z_{c}\right)$. The barycentric coordinate of each group is obtained based on Figure 1: 


$$
\begin{aligned}
& \left(x_{a}, y_{a}, z_{a}\right)=\left(0,0,-\left(\frac{H}{2}+d_{1}+\frac{d_{2}}{2}+L_{G} \cdot \sin \sigma\right)\right), \\
& \left(x_{b}, y_{b}, z_{b}\right)=\left(0,0,-\left(\frac{H}{2}+d_{1}+d_{2}+2 \cdot L_{G} \cdot \sin \sigma+d_{3}\right)\right), \\
& \left(x_{c}, y_{c}, z_{c}\right)=\left(R \cdot \cos \theta_{a}, R \cdot \sin \theta_{a},-\left(\frac{H}{2}+d_{1}+d_{2}+2 \cdot L_{G} \cdot \sin \sigma+d_{3}+\frac{h}{2}\right)\right) \text {, } \\
& G_{0}=(x, y, z)=\frac{\sum_{i=a}^{c}\left(M_{i} G_{i}\right)}{\sum_{i=a}^{c} M_{i}}, \\
& \sum_{i=a}^{c} M_{i}=M_{G}=M_{a}+M_{b}+M_{c} \\
& \left\{\begin{array}{l}
x=\frac{x_{c} \cdot R \cdot \cos \theta_{a}}{M_{G}}, \\
y=\frac{y_{c} \cdot R \cdot \sin \theta_{a}}{M_{G}}, \\
z=\left[-z_{a} \cdot\left(\frac{H}{2}+d_{1}+\frac{d_{2}}{2}+L_{G} \cdot \sin \sigma\right)-z_{b} \cdot\left(\frac{H}{2}+d_{1}+d_{2}+2 \cdot L_{G} \cdot \sin \sigma+d_{3}\right) .\right.
\end{array}\right.
\end{aligned}
$$

The forest chassis structure is relatively regular, so the corresponding center of gravity can be calculated. To reduce the variables in the theoretical calculation, it is reasonably simplified into two parts, frame and legs. In addition, assuming that all chassis are of the same homogeneous material, each part's geometric center is its barycenter. The weight of the legs is ignored since it is relatively small compared with the whole chassis. Thus, the barycenter is determined by the frame and barycenter balanced structure, and the barycenter of the frame coincides with its geometric center.

Let the barycentric coordinate of the forestry chassis is $G(x, y, z)$, which can be obtained based on formula (3):

$$
\left\{\begin{array}{l}
x=\frac{\left(M_{0} \cdot x_{0}+M_{G} \cdot x_{G}\right)}{M} \\
y=\frac{\left(M_{0} \cdot y_{0}+M_{G} \cdot y_{G}\right)}{M} \\
z=\frac{\left(M_{0} \cdot z_{0}+M_{G} \cdot z_{G}\right)}{M}
\end{array}\right.
$$

where $M=M_{0}+M_{G}$.

Based on the above mathematical analysis and the motion parameters in Table 2, the barycenter varying law of BAM and the chassis with different parameter values are analyzed. On the basis of structure, the rotation range of BAM is 0 to 360 degrees. To avoid repetition, the chassis frame is divided into four parts, and the quarter section is selected as the analysis area, as shown in Figure 2(b). 1, 2, 3, and 4 represent four support points of chassis, 1,2, and 3 are in the supporting phase and 4 is in the swing phase. Namely, the motion range of counterweight is 0 to 90 degrees.
2.3. Control Method of Chassis Barycenter. Compared with the flat terrain, it is more difficult for the chassis to walk on the slope condition. The reason is that the projection of barycenter on the support surface is not on the same horizontal plane as the support points of each leg, and the horizontal projection area of the support triangle becomes smaller so that the stability margin $\left(S_{m}\right)$ becomes lower. $S_{m}$ should be greater than 0 , and it is better to reach the maximum $S_{m \max }$. More significant $S_{m}$ conduces to improve the chassis stability. Based on this, a method is proposed to increase $S_{m}$.

The initial state of forestry chassis on a slope is shown as Figure 3, and the frame is parallel to the inclined plane. It is assumed that the barycenter coincides with the origin of the frame coordinate system. As the chassis moves in a static gait, each leg is raised alternately. The barycenter's projection on the slope surface will change continuously along with the support triangles formed by the other three supporting legs. Projection of barycenter falls in the back of projection area. What's worse, projection of barycenter will fall outside the support polygon with large inclination. Thus, the barycenter's projection is close to the limit position, which makes the chassis stability extremely poor, or during the gait transformation, especially when the hind leg is in the swing phase, projection of barycenter falls outside the polygon, causing the chassis to tip over. Therefore, it is a great idea to adjust the barycenter to ensure that projection of barycenter is always in the support triangle and increase the stability margin as much as possible.

The intersection point of two diagonal lines is taken as the origin to define the barycenter projection's stable area. As long as the barycenter's projection falls at the inner of the supporting triangle, the chassis gets the maximum stability margin. Thus, the chassis barycenter translates $R_{\max }$ and 
TABle 2: Parameter value of balance mechanism.

\begin{tabular}{lccc}
\hline Parameters & $R(\mathrm{~mm})$ & $\theta_{a}\left(^{\circ}\right)$ & $\sigma\left(^{\circ}\right)$ \\
\hline 1 & $0-120$ & 0 & 0 \\
2 & 0 & $0-90$ & 0 \\
3 & 0 & $0-90$ & $0-90$ \\
4 & $0-120$ & 0 & $0-90$ \\
5 & $0-120$ & $0-90$ & 0 \\
6 & 0 & 0 & $0-90$ \\
7 & $0-120$ & $0-90$ & $0-90$ \\
8 & $0,60,120$ & 0 & 0 \\
\hline
\end{tabular}
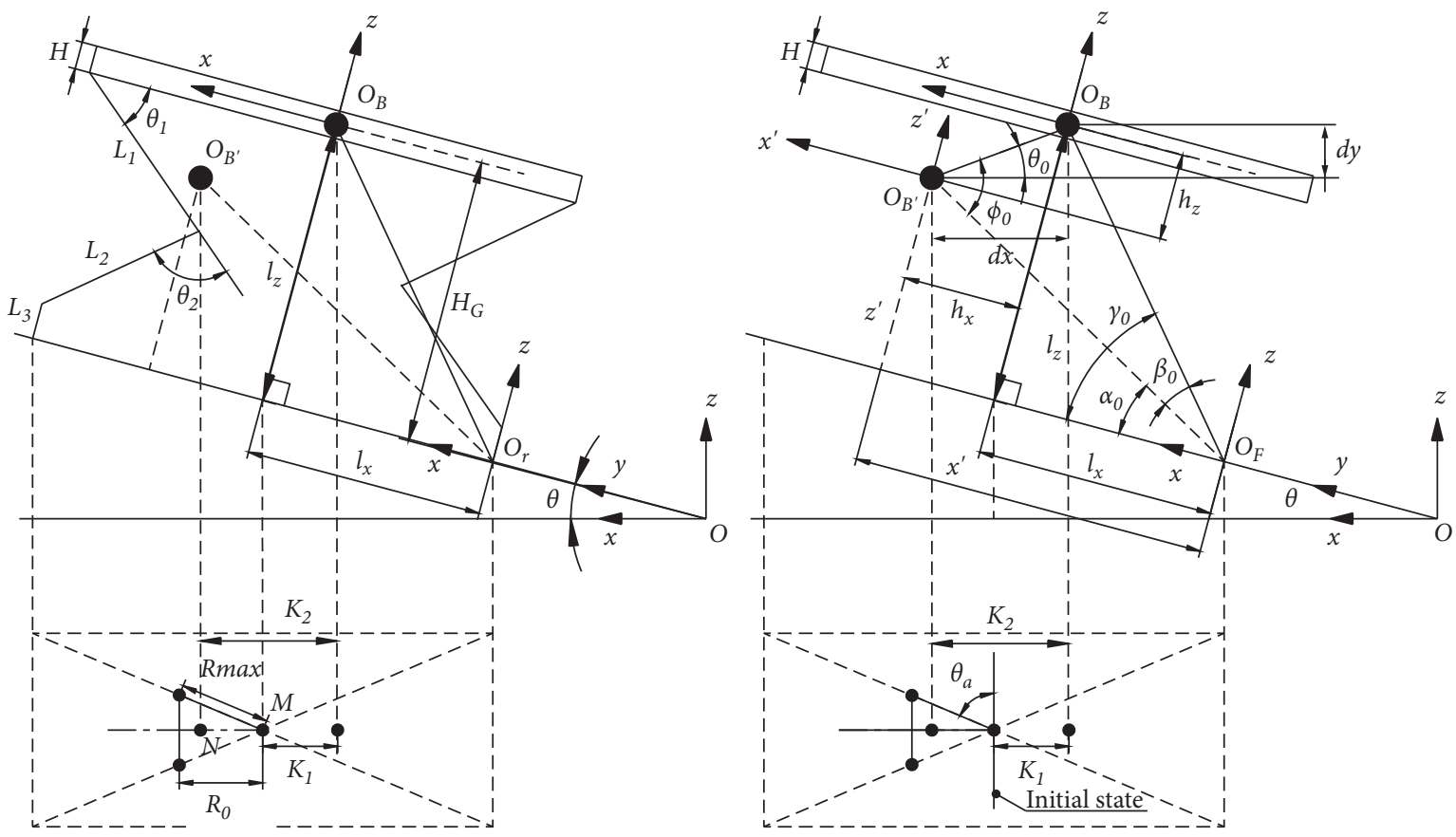

Figure 3: Projection of barycenter and barycenter adjustment.

rotates $\theta_{a}$. Namely, the horizontal forward displacement of chassis barycenter is $R_{0}$.

Let the translation of counterweight is $R$, and rotation is $\theta_{a}$ and vertical drop $h_{z}$, correctly, parameters of BAM can ensure the chassis barycenter's projection falls close to the triangle inner, improving the stability margin. As shown in Figure 3, $M$ is the intersection of diagonal lines of chassis support points. $K_{1}$ is the distance between $M$ and the projection of the chassis barycenter. $K_{2}$ is the displacement that the chassis barycenter moves forward with the adjustment of BAM. Based on the stability margin, the stability condition of chassis motion is as in formula (5):

$$
K_{1}<K_{2} \text {. }
$$

The above conditions can ensure that $S_{m}$ is more significant than zero, but it may be inadequate. To increase $S_{m}$, the stability condition of chassis motion can be further determined as

$$
K_{1}<K_{2} \leq K_{1}+R_{0}
$$

Figure 3 shows the chassis barycenter before and after BAM adjustment. $O_{B}$ is the initial position of the chassis barycenter, and $O_{B}^{\prime}$ is the position after adjustment.

The position coordinates of $O_{B},\left(x_{0}, z_{0}\right)$ in the reference coordinate system $\left\{O_{F}\right\}$ is as in

$$
\left\{\begin{array}{l}
x_{0}=l_{x} \\
z_{0}=l_{z}
\end{array}\right.
$$

In the initial state, the vertical distance from the chassis barycenter to the inclined plane can be calculated according to the kinematics, as shown in

$$
l_{z}=L_{1} \cdot \sin \theta_{1}+L_{2} \cdot \sin \left(\theta_{1}+\theta_{2}\right)+L_{3}
$$

After moving forward, position coordinates of $O_{B}^{\prime}$, $\left(x^{\prime}, z^{\prime}\right)$ in the reference coordinate system $\left\{O_{F}\right\}$ is as in

$$
\left\{\begin{array}{l}
x^{\prime}=l_{x}+h_{x}=l_{x}+R \cdot \cos \theta_{a}, \\
z^{\prime}=l_{z}-h_{z} .
\end{array}\right.
$$

Thus, $K_{1}$ and $K_{2}$ can be calculated as follows: 


$$
\begin{aligned}
K_{1}= & \left(L_{1} \cdot \sin \theta_{1}+L_{2} \cdot \sin \left(\theta_{1}+\theta_{2}\right)+L_{3}\right) \cdot \sin \theta \\
K_{2}= & \sqrt{h_{x}^{2}+h_{z}^{2}} \cdot \cos \left[\left(\arccos \frac{{\overline{O_{B B}^{\prime}}}^{2}+{\overline{O_{B F}^{\prime}}}^{2}-{\overline{O_{B F}}}^{2}}{2 \cdot{\overline{O_{B B}^{\prime}}}_{\overline{O_{B F}^{\prime}}}^{\prime}}\right)\right. \\
& -\theta-\arctan \left(\frac{l_{z}-h_{z}}{l_{z}+h_{x}}\right) .
\end{aligned}
$$

By substituting the above results in formula (6), we can get the following relation:

$$
\begin{aligned}
& {\left[L_{1} \cdot \sin \theta_{1}+L_{2} \cdot \sin \left(\theta_{1}+\theta_{2}\right)+L_{3}\right] \cdot \sin \theta<\sqrt{h_{x}^{2}+h_{z}^{2}}} \\
& \quad \cdot \cos \left[\left(\arccos \frac{{\overline{O_{B B}^{\prime}}}^{2}+{\overline{O_{B F}^{\prime}}}^{2}-{\overline{O_{B F}}}^{2}}{2 \cdot \overline{O_{B B}^{\prime}} \cdot \overline{O_{B F}^{\prime}}}\right)-\theta-\arctan \left(\frac{l_{z}-h_{z}}{l_{z}+h_{x}}\right) \leq\left(L_{1} \cdot \sin \theta_{1}+L_{2} \cdot \sin \left(\theta_{1}+\theta_{2}\right)+L_{3}\right) \cdot \sin \theta+\frac{\sqrt{W^{2}+L^{2}}}{6}\right.
\end{aligned}
$$

2.4. Gait and Trajectory Planning. The forest chassis needs to complete the under forest operation in a narrow space on slope terrain. The chassis requires to keep stable operation, and it is not necessary for the chassis to move at mediumhigh speed due to the forest environment.

The route of chassis motion is idealized as a straight line here. Based on the fact that the barycenter is as close as possible to the inner of the supporting triangle, the barycenter adjustment is added to the chassis' steady static gait to improve the stability margin. We take the six-leg stepping sequences of leg 2 swinging first as an example. The number and amount of barycenter adjustment are analyzed to reduce the impact of barycenter adjustment on the motion system's stability. Moreover, the distance of barycenter adjustment in a cycle is allocated to each phase to achieve a slight distance adjustment in a single movement. The shaking caused by inertial force due to the large movement of barycenter and the risk of robot overturning can be reduced. The time required for each execution phase is fully used to make the barycenter moving trajectory smooth and the motion more efficient. Therefore, the leg swing sequence is selected as 2-14-3 for semi-intermittent continuous gait planning. The gait sequence is shown in Figure 4 in which the solid line part represents the support phase. The sketch of gait and barycenter adjustment is shown in Figure 5.

Based on the compound cycloid, a modified trajectory suitable for forest chassis is planned here. As shown in Figure 6, the chassis' front leg trajectory is planned in the shoulder joint coordinate system. The compound cycloid is adopted to plan the swing phase trajectory $\widehat{P_{F F^{\prime}}}$, and the linear equation is used for support phase trajectory $\overline{P_{F F^{\prime}}}$.

$T$ is when four legs complete a movement, and $t$ is the time variable in one cycle $(0 \leq t \leq T)$. According to the chassis gait, $T_{s w}$ (swing phase) $=T / 4, T_{s t}$ (support phase) $=(3 / 4) T$. In one-fourth cycle $\left(0 \leq t \leq T_{s w}\right)$, the foot swings from $P_{F}$ to $P_{F}^{\prime}$ along the moving direction. The trajectory cannot have sudden change of velocity and acceleration in the conversion process of support phase and swing phase to keep stable movement. Thus, the limit conditions are as in

$$
\left\{\begin{array} { l } 
{ x _ { s w } ^ { 2 } ( 0 ) = - \frac { S } { 2 } , } \\
{ x _ { s w } ^ { 2 } ( \frac { T } { 4 } ) = 0 , } \\
{ x _ { s w } ^ { 2 } ( 1 ) = \frac { S } { 2 } , }
\end{array} \left\{\begin{array} { l } 
{ \dot { x } _ { s w } ^ { 2 } ( 0 ) = 0 , } \\
{ \dot { x } _ { s w } ^ { 2 } ( \frac { T } { 4 } ) = 0 , } \\
{ \dot { x } _ { s w } ^ { 2 } ( 1 ) = 0 , }
\end{array} \left\{\begin{array}{l}
\ddot{x}_{s w}^{2}(0)=0 \\
\ddot{x}_{s w}^{2}\left(\frac{T}{4}\right)=0 \\
\ddot{x}_{s w}^{2}(1)=0 .
\end{array}\right.\right.\right.
$$

In motion direction, the foot trajectory of swing phase adopts the cycloid equation as shown in equations (13) and (14) that are obtained after integration, and equation (15) is obtained after integrating twice:

$$
\begin{aligned}
& \ddot{x}_{s w}^{2}(t)=A_{1} \sin (4 \pi t) \quad 0 \leq t \leq T_{s w}, \\
& \dot{x}_{s w}^{2}(t)=-\frac{A_{1}}{4 \pi} \cos (4 \pi t)+C_{1} \quad 0 \leq t \leq T_{s w},
\end{aligned}
$$




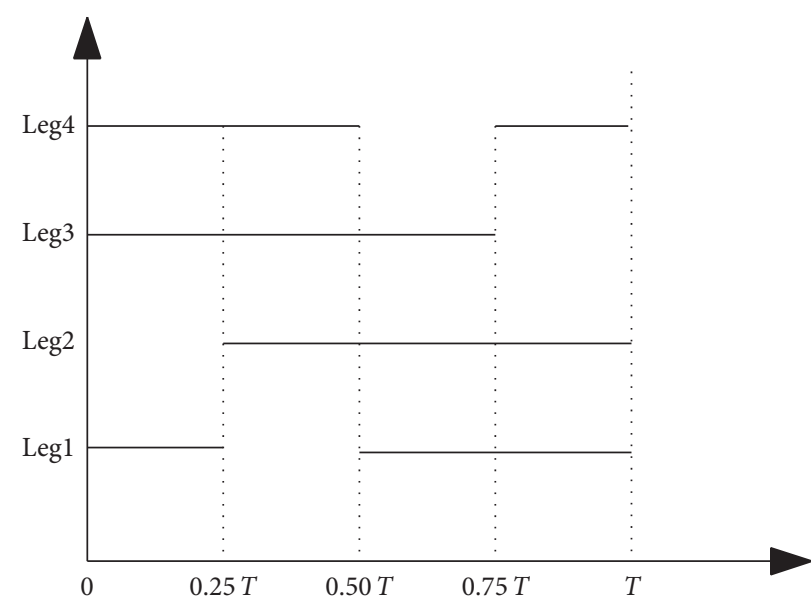

FIGURE 4: Semi-intermittent continuous gait sequence.
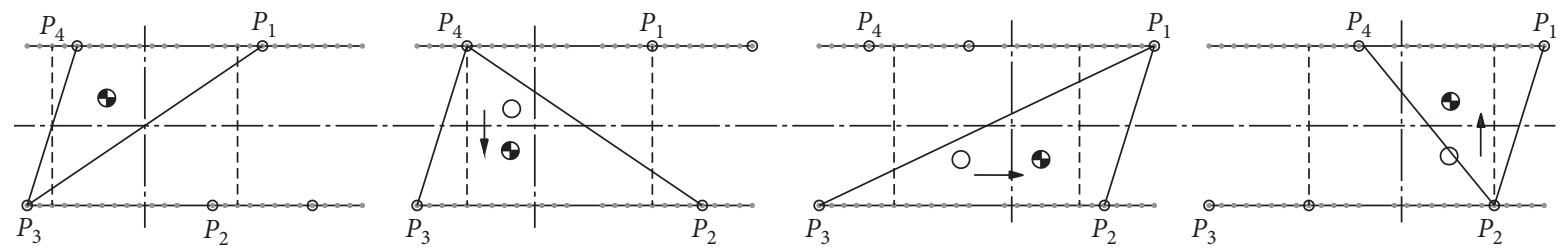

Figure 5: Sketch of barycenter adjustment.

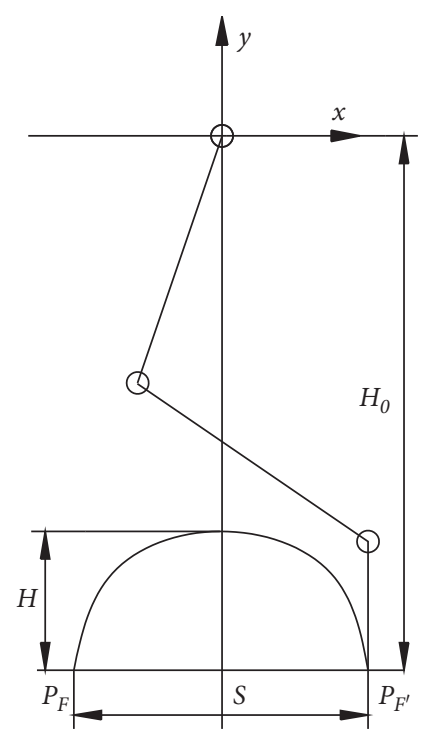

Figure 6: Trajectory planning of front leg.

$$
x_{s w}^{2}(t)=-\frac{A_{1}}{(4 \pi)^{2}} \sin (4 \pi t)+C_{1} t+C_{2} \quad 0 \leq t \leq \frac{T}{4} .
$$

$A_{1}, C_{1}$, and $C_{2}$ are obtained by substituting the limiting conditions into the formula, and then the formula of foot trajectory along motion direction in the $x$-axis is obtained as in

$$
\begin{aligned}
x_{s w}^{2}(t)= & -\frac{S}{n \pi} \sin (n \pi t)+S t-\frac{S}{2}=S\left(t-\frac{1}{n \pi} \sin (n \pi t)\right) \\
& -\frac{S}{2} \quad 0 \leq t \leq T_{s w} .
\end{aligned}
$$


To determine the suitable value of $n$, curves of $x_{s w}^{2}(t)$ with $n=2,4,6,8$ are plotted in Matlab; Figure 7 shows that the trajectory is smoothest as $n=2$.

For the y-axis, the leg motion in the swing phase can be divided into two processes, and the foot lifts from zero position and reach the highest point when $t=T_{s w} / 2$, then lowers to ground. At $t=T_{s w} / 2$, the foot position is determined as $y_{s w}^{2}\left(T_{s w} / 2\right)=-H_{0}+H$. Also, the transition of the foot end from the swing phase to the supporting phase needs to satisfy the continuity and acceleration of the equation, and the restriction condition is as in

$$
\left\{\begin{array} { l } 
{ y _ { s w } ^ { 2 } ( 0 ) = - H _ { 0 } , } \\
{ y _ { s w } ^ { 2 } ( \frac { T _ { s w } } { 2 } ) = - H _ { 0 } , } \\
{ y _ { s w } ^ { 2 } ( 1 ) = - H _ { 0 } , } \\
{ \dot { y } _ { s w } ^ { 2 } ( \frac { T _ { s w } } { 2 } ) = 0 , } \\
{ \dot { y } _ { s w } ^ { 2 } ( 1 ) = 0 , }
\end{array} \left\{\begin{array}{l}
\dot{y}_{s w}^{2}(0)=0, \\
\ddot{y}_{s w}^{2}\left(\frac{T_{s w}}{2}\right)=0, \\
\ddot{y}_{s w}^{2}(1)=0 .
\end{array}\right.\right.
$$

According to the motion order, the leg lifting trajectory from zero position to the highest point is first planned, and equation (18) is selected as the acceleration equation. The parameters are obtained through the integral formula, and the foot lifting trajectory is as in

$$
\begin{aligned}
& \ddot{y}_{s w}^{2}(t)=A_{2} \sin (n \pi t) \quad 0 \leq t \leq \frac{T_{s w}}{2}, \\
& y_{s w}^{2}(t)=2 H\left(t-\frac{1}{n \pi} \sin n \pi t\right)-H_{0} \quad 0 \leq t \leq \frac{T_{s w}}{2} .
\end{aligned}
$$

The trajectories of foot falling and lifting are symmetrical in the $y$-direction. The foot falling trajectory is obtained as in

$$
y_{s w}^{2}(t)=2 H\left(1-t+\frac{1}{n \pi} \sin n \pi t\right)-H_{0} \quad \frac{T_{s w}}{2} \leq t \leq T_{s w} .
$$

The curve of $y_{s w}^{f}(t)$ shows that when $n=4$, the curve is most stable. Therefore, the foot trajectory equation of the foreleg in the swing phase is as in

$$
\left\{\begin{array}{l}
x_{s w}^{2}(t)=S\left(t-\frac{1}{2 \pi} \sin (2 \pi t)\right)-\frac{S}{2} \quad 0 \leq t \leq T_{s w}, \\
y_{s w}^{2}(t)=\left\{\begin{array}{l}
2 H\left(t-\frac{1}{4 \pi} \sin 4 \pi t\right)-H_{0} \quad 0 \leq t \leq \frac{T_{s w}}{2}, \\
2 H\left(1-t+\frac{1}{4 \pi} \sin 4 \pi t\right)-H_{0} \quad \frac{T_{s w}}{2} \leq t \leq T_{s w} .
\end{array}\right.
\end{array}\right.
$$

In the supporting phase, the foot does not move on the ground, while the body moves forward relative to the ground, so the foot slides from $P_{F}$ ' to $P_{F}$, resulting in a straight line. Based on the gait, the constraints are as follows:

$$
\left\{\begin{array}{l}
x_{s t}^{2}(1)=\frac{S}{2} \\
x_{s t}^{2}(T)=-\frac{S}{2}
\end{array}\right.
$$

The foot has no displacement in the y-axis, so $y_{s t}^{2}=H_{0}$ is a fixed value. The trajectory equation along the forward direction of the $x$-axis is a linear function, as shown in

$$
x_{s t}^{2}(t)=S\left(a_{0} t+a_{1}\right) \quad 1 \leq t \leq T .
$$

The formula of trajectory in the supporting phase is obtained through the restriction condition (24):

$$
\left\{\begin{array}{l}
x_{s t}^{2}(t)=S\left(-\frac{1}{3} t+\frac{5}{6}\right), \\
y_{s t}^{2}(T)=-H_{0}, 1 \leq t \leq T .
\end{array}\right.
$$

Take the following parameters as examples: $T=4 s$, step length $S=200 \mathrm{~mm}$, lifting height $H=60 \mathrm{~mm}$, and the distance between shoulder joint to foot $H=360 \mathrm{~mm}$. In the shoulder coordinate system, the compound cycloid trajectory of the foot is shown in Figure 8.

Figure 9 shows the displacement, velocity, and acceleration curves along the $x$-axis. Figure 10 shows the displacement, velocity, and acceleration curves along the $y$-axis. The results show that in the swing phase to support phase and swing phase to support phase, the foot can avoid dragging on the ground, and at the moment of transition, the speed and acceleration change smoothly without the mutation.

\section{Results}

3.1. Barycenter Variation Curves. Representative barycenter variation curves according to parameters are shown in Figure 11. The results show that when the counterweight shifts $120 \mathrm{~mm}$ without rotation or falling, the barycenter deviates with the counterweight in the same direction $(x)$ and the offset is $100 \mathrm{~mm}$. The lateral $(y)$ and vertical $(z)$ do not change. However, if the counterweight does not shift, the rotation and vertical motion parameters rotate from $0^{\circ}$ to $90^{\circ}$, and the barycenter of BAM gradually decreases along the $z$-direction, while no variation in the $x$ - and $y$-direction. 

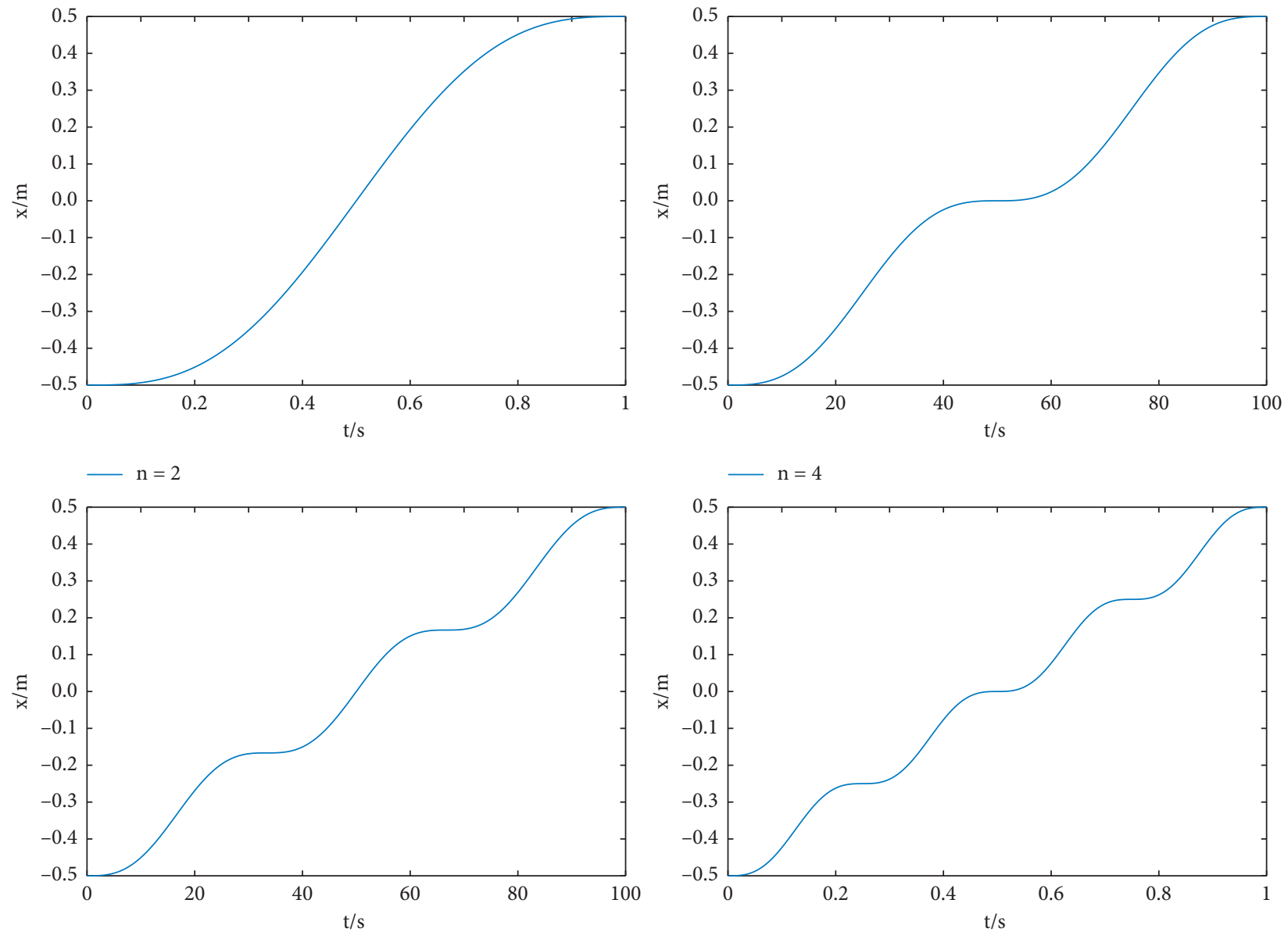

$-\mathrm{n}=6$

- $\mathrm{n}=8$

Figure 7: $x_{s w}^{2}(t)$ varies with $n$.

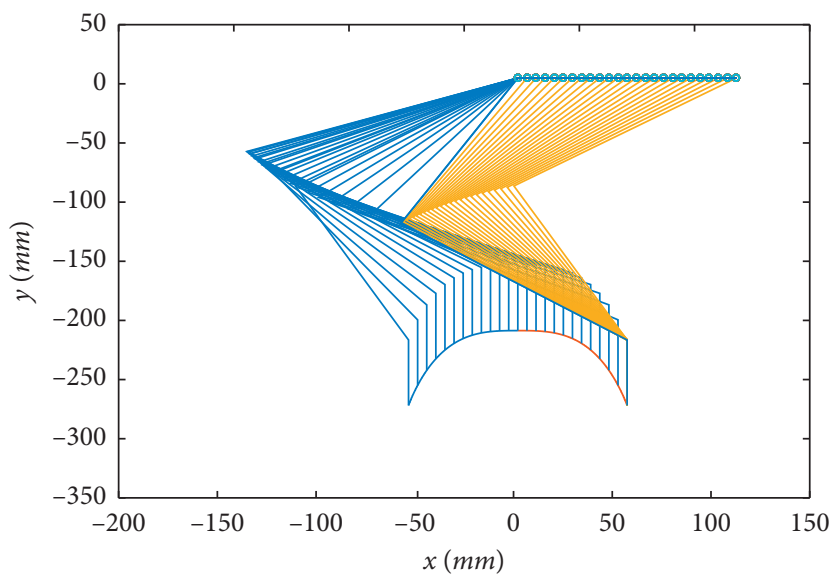

Figure 8: Foot trajectory of one front leg.

If the counterweight shifts and descends without rotation, the barycenter moves along the $x$-direction and lower. As the counterweight moves with three parameters concurrently, the barycenter is constantly lower while varying in the $x$-and $y$-direction. Finally, three groups of fixed translation values are taken; it can be seen that the longer the translation, the greater the barycenter offset. However, as the counterweight rotates, the barycenter offset in the $x$-direction gradually decreases to 0 , while the offset in the $y$-direction gradually increases to the maximum.

Figure 11(d) clearly shows that the variation of barycenter in the $x$ - and $y$-direction intersects when the 

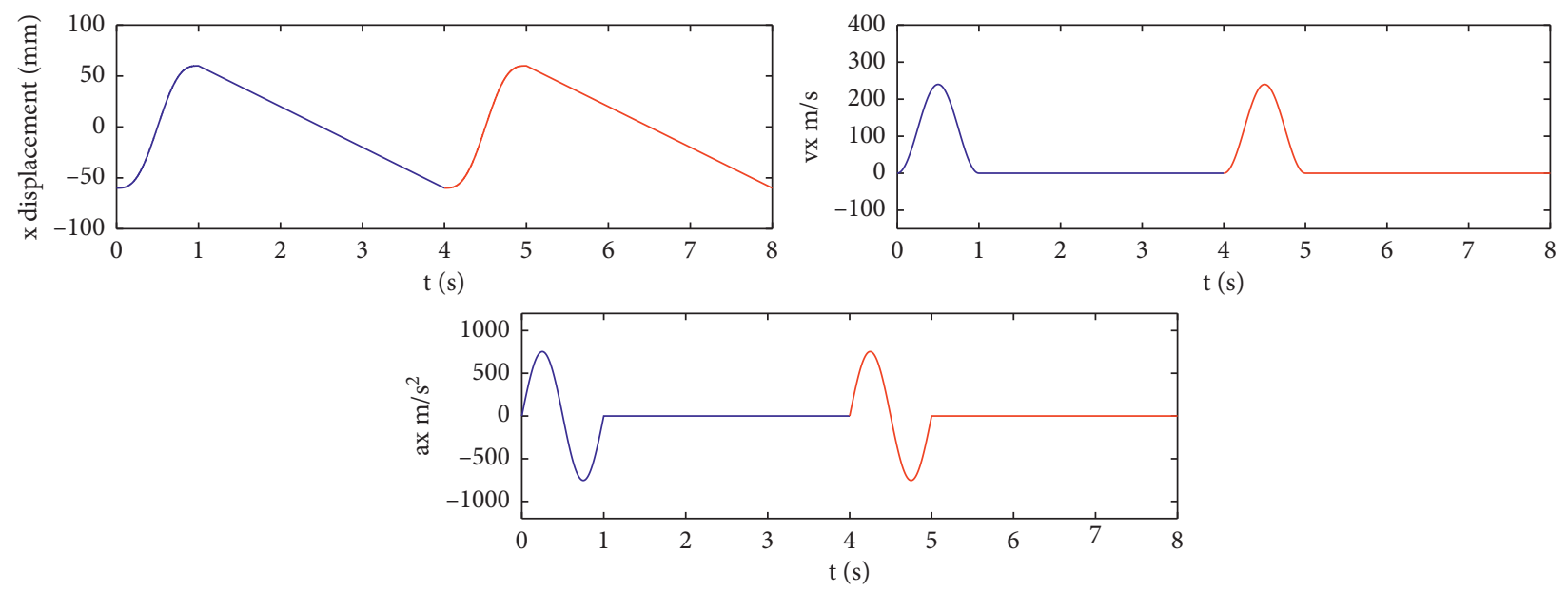

Figure 9: Foot displacement, velocity, and acceleration curves in $x$-direction.
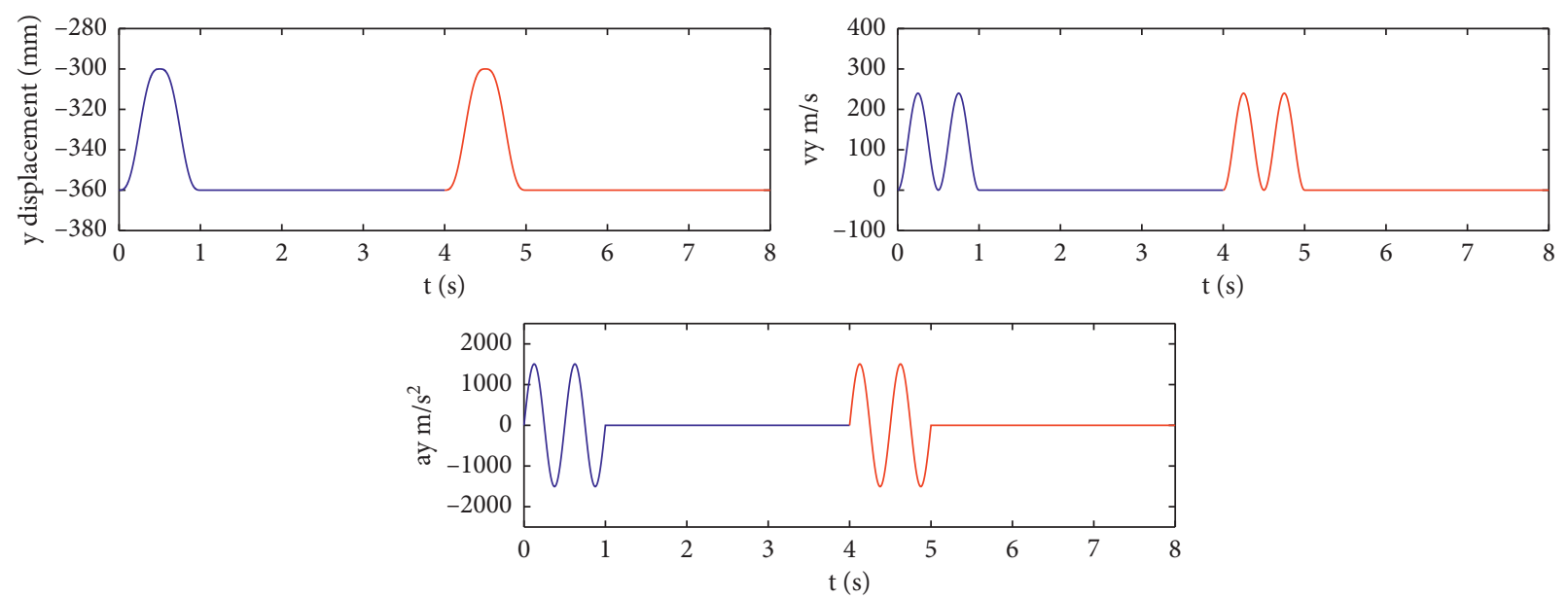

FIGURE 10: Foot displacement, velocity, and acceleration curves in the $y$-direction.

counterweight is closest to the inner of the supporting triangle. At the same time, if the vertical displacement of the counterweight is the largest, namely, the three parameters are $R=120 \mathrm{~mm}, \theta_{a}=45^{\circ}, \sigma=90^{\circ}$, the barycenter of BAM reaches the maximum adjustment, bringing the same effect on chassis barycenter, to improve the chassis stability.

3.2. Simulation. According to the above results, it is evident that BAM can adjust the chassis barycenter, but its motion characteristics during one adjustment cycle are still unclear. Therefore, in this section, Adams software is adopted to simulate the motion under the static gait. The virtual prototype built and assembled in Solid Edge with the same physical parameters (Table 3), and BAM is simplified for the convenience of simulation. Then, the $x$ - $t$ file is imported into Adams for simulation. The adjustment parameters of BAM are set as $R=100 \mathrm{~mm}, \theta_{a}=45$ degrees, and $\sigma=50$ degrees. The simulation process is shown in Figure 12.

The simulation results of BAM adjustment and chassis climbing are shown in Figure 13. Climbing is the whole process of the chassis moving from the flat to the slope.
During the moving process, the barycenter of the chassis frame fluctuates in a small range and keeps stable, as shown in Figure 13(a).

Figure 13(b) shows the result of one adjustment cycle of the BAM. The motion initialization includes three processes, and the counterweight rotates 45 degrees first, then it shifts, and finally it drops by about $150 \mathrm{~mm}$. During the rotation, the counterweight stays in the center, without variation in three directions. With translational motion, the barycenter moves to the support triangle. Finally, the barycenter drops significantly with the links moving from horizontal to vertical. Then, BAM rotates 90 degrees with the chassis step, which shows that the barycenter curve of BAM varies regularly.

As a result, it can be seen that the BAM can adjust the barycenter position by changing parameters, so that the stability margin can be improved, contributing to the stability of the chassis in slope conditions.

3.3. Experiment. The chassis platform is shown in Figure 14(a), consisting of servo motors, linear actuators, and BAM, and the parameters are presented in Table 3. The 


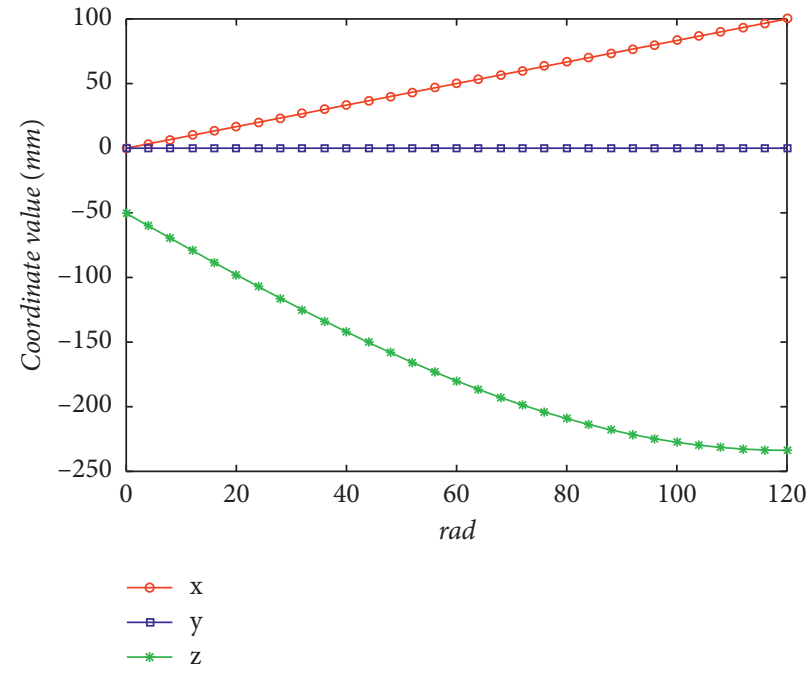

(a)

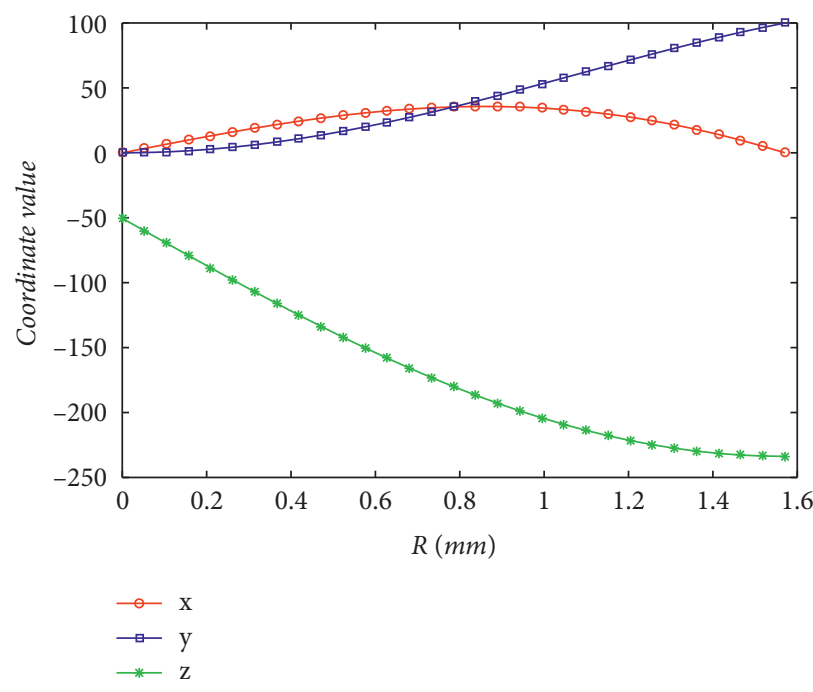

(c)

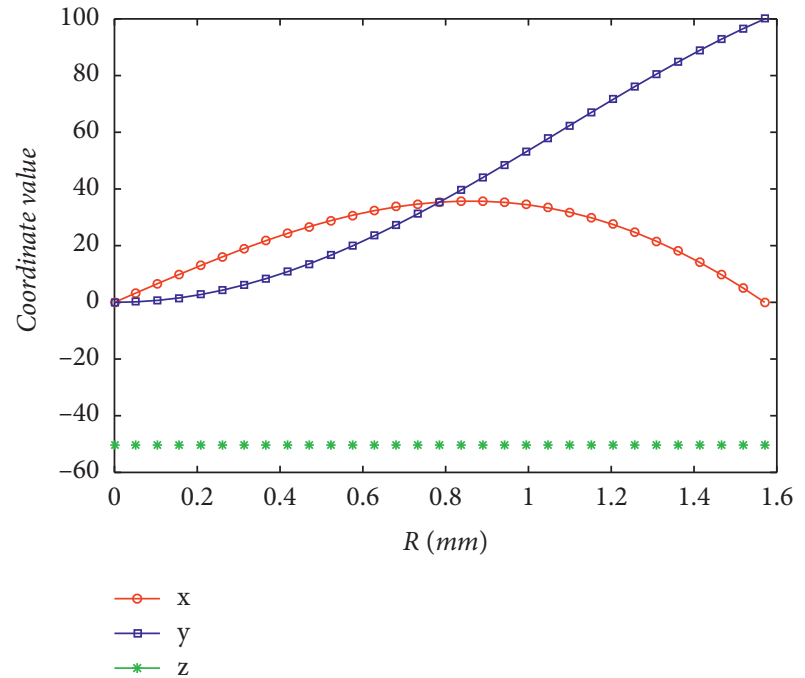

(b)

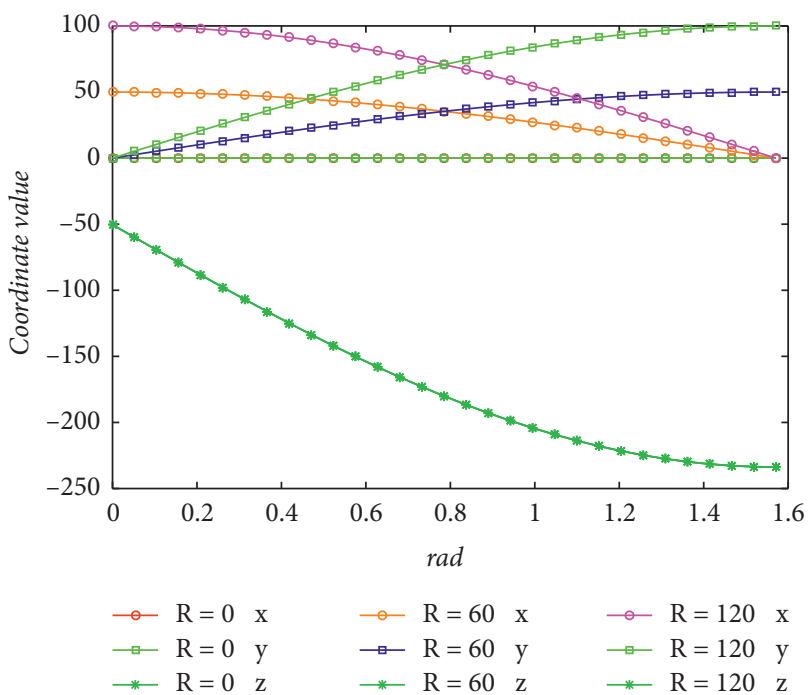

(d)

FiguRE 11: Barycenter varies with parameters. (a) $R=0-120, \theta_{a}=0^{\circ}, \sigma=0-90^{\circ}$; (b) $R=0-120, \theta_{a}=0-90^{\circ}, \sigma=0^{\circ}$; (c) $R=0-120, \theta_{a}=0-90^{\circ}$, $\sigma=0-90^{\circ}$; and (d) $R=0,60,120, \theta_{a}=0-90^{\circ}, \sigma=0-90^{\circ}$.

TABLE 3: Parameters of chassis prototype.

\begin{tabular}{|c|c|c|c|c|c|}
\hline Parameter & Body & Link 1 & Link 2 & Link 3 & BAM \\
\hline Length $(\mathrm{mm})$ & $690 \times 455 \times 8$ & 176 & 168 & 70 & \\
\hline Mass (Kg) & 7 & 0.7 & 0.6 & 0.2 & 4 \\
\hline Material & & & um alloy & & \\
\hline
\end{tabular}

hardware configuration of BAM mainly includes three motors drives, two sets of rails and sliders with a length of $240 \mathrm{~mm}$, a screw, a precision bearing, and a counterweight. Three drives controlled through Arduino are used to drive motors separately. Figure 14(b) shows the control hardware configuration. Considering that the motor shaft will always bear load during movement, a precision bearing is used for connecting that can bear the load from the lower parts and avoid damage to the shaft. The precision linear rail and slide adopt a rolling friction model, which significantly reduces the friction coefficient, and it can bear the load of counterweight. Additionally, the counterweight-driven way is specifically designed that simplifies the structure by using the motor as a part of the counterweight. The counterweight is driven to raise or lower through a screw, convenient for accurate driving based on pitch and lead. 

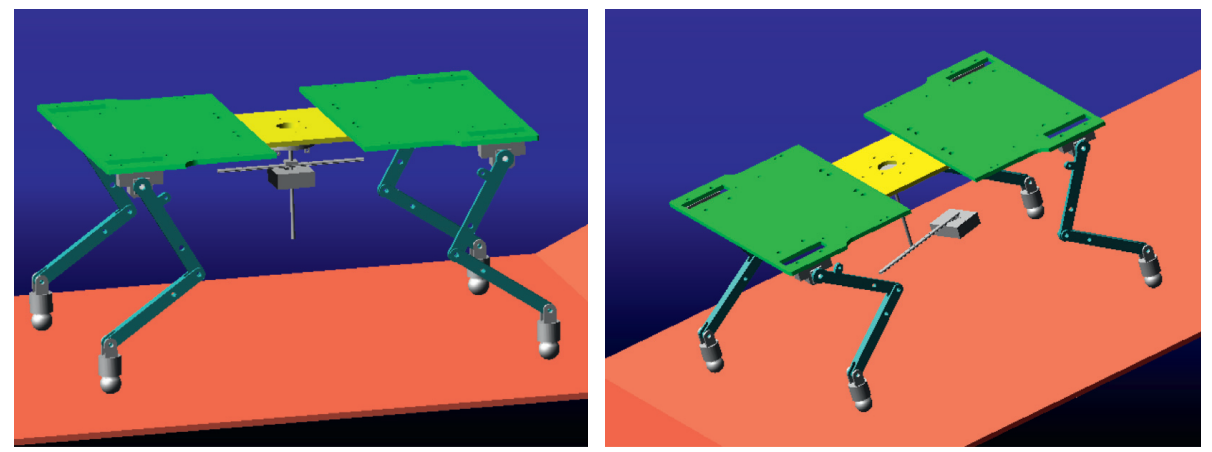

Figure 12: Model of chassis with BAM used in the simulation.
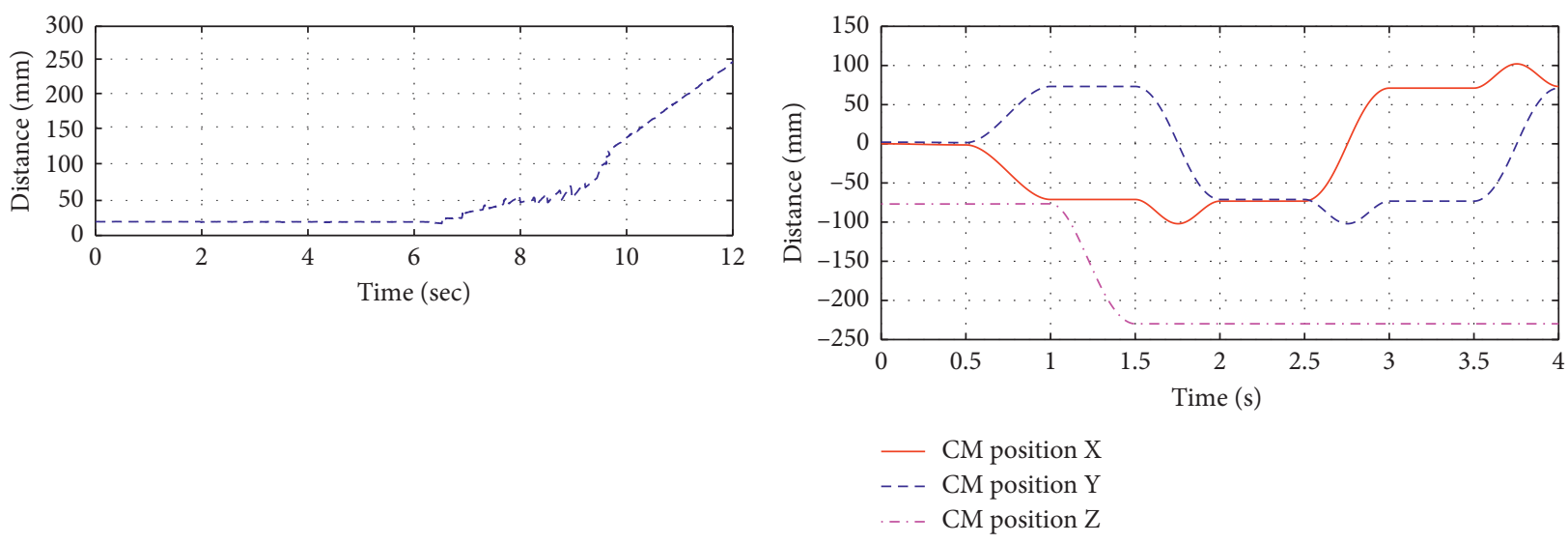

(a)

(b)

Figure 13: (a) Centroid curve of the chassis frame. (b) Centroid curve of counterweight.

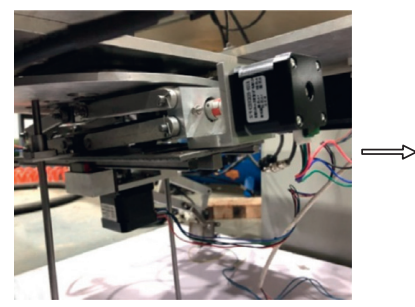

(a)

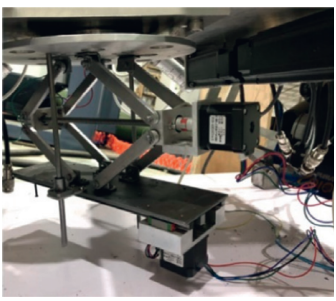

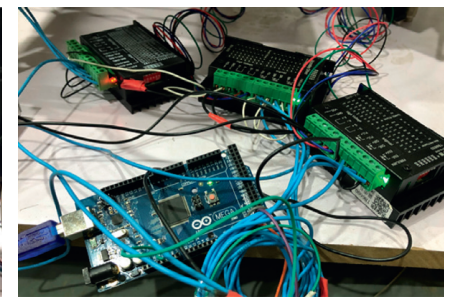

(b)

Figure 14: (a) State of BAM before and after adjustment. (b) Control hardware configuration.

Other experimental equipment is as follows: the Arduino, a laptop, 6 sets of pressure sensors (model: RFP803), and matching conversion module (model: RFPZHII) as shown in Figure 15(a), and connecting lines. The output data of the conversion module are obtained as voltage. Figure 15(b) shows the duration curve of the highand low-voltage output of the conversion module. The power supply voltage of the conversion module is $5 \mathrm{~V}$. When no load is applied, the module outputs a high voltage. At this time, the measured voltage is $1024 \mathrm{mV}$. With the increase in load, the input voltage of the module decreases. The test data result is $280 \mathrm{mV}$, which is the maximum load.

Stability experiments are carried out on simplified slope conditions, which are manually made by hand, as shown in
Figure 16. Slope conditions of $0,5,10,15$, and 20 degrees are created, and the slope is adjusted after each experiment, avoiding the impact of the last test. Each test is done more than three times.

Two sets of comparative tests are conducted according to the following procedures. Under slope working conditions, the chassis is easy to roll over when the rear leg is in swing phase. Due to the symmetrical chassis structure, the pressure value change is only tested when the left rear leg is raised and the other three legs are in the supporting phase. The first set of pressure values is measured with a standard chassis, as shown in Figure 16(a). Pressure values of the three supporting legs are obtained under the above slope conditions to be a control group. For comparison, the other set of pressure 


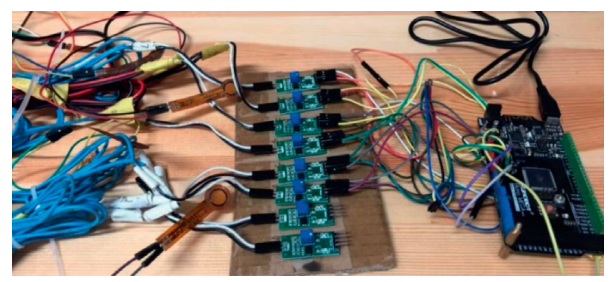

(a)

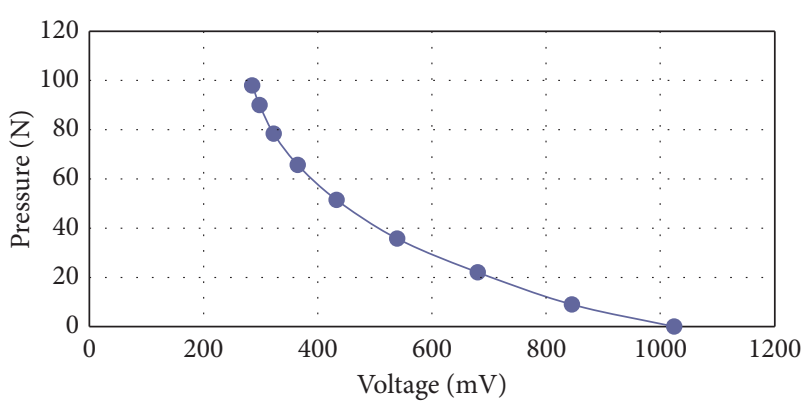

(b)

Figure 15: (a) Hardware for data reading. (b) Calibration of force and voltage.

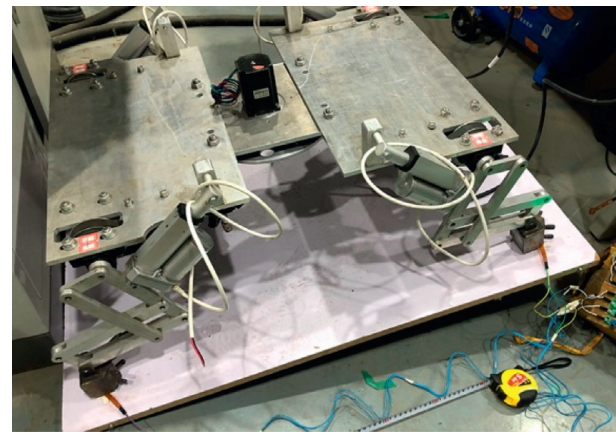

(a)

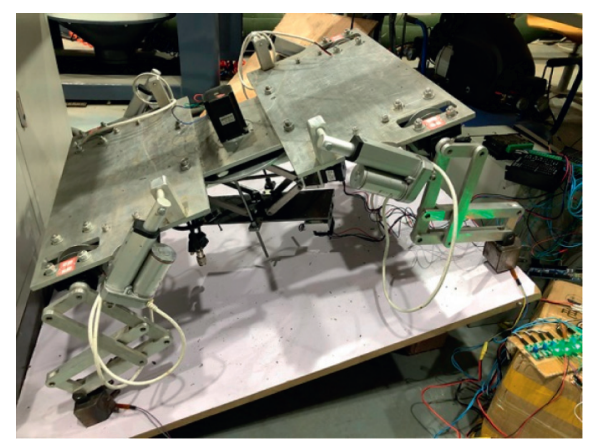

(b)

Figure 16: Pressure test of (a) chassis and (b) chassis installed BAM.

values with $F C$ and $B A M$ is measured and recorded to be test group, and the three parameters are taken as $R=120 \mathrm{~mm}$, $\theta_{a}=45$ degrees, and $\sigma=45$ degrees.

The results are shown in Table 4, and the standard chassis can be stable on a slope of 5 degrees, while the chassis installed barycenter adjustable mechanism can be stable on the slope of 15 degrees.

The stability of a standard chassis and forestry chassis with BAM is compared by analyzing two groups of plantar pressure data. It shows that the plantar pressure of standard chassis' leg 2 on the slope of 5 degrees is about $10 \mathrm{~N}$ and that of leg 3 is about $70 \mathrm{~N}$. The contrastive value is $39 \mathrm{~N}$ for leg 2 of the chassis with BAM and $77 \mathrm{~N}$ for leg 3 on the same slope.

The plantar pressure of the chassis with BAM is more significant than that of the standard chassis. The self-weight of the BAM is one reason to slightly increase the plantar pressure. The key reason is that the counterweight adjustment makes the barycenter of the chassis move to the support triangle, increasing the pressure of foreleg 2 to improve the chassis stability. As the slope becomes steeper, the plantar force of leg 3 increases and that of leg 2 decreases. On the slope of 15 degrees, the pressure of foreleg 2 is about $14 \mathrm{~N}$ and that of leg 3 is about $83 \mathrm{~N}$. The results reflect that BAM is conductive to the chassis stability under slope working conditions.

\section{Discussion}

Our idea of chassis with BAM focuses on improving chassis stability by adjusting suitable barycenter positions. The
TABLE 4: Test conditions and values.

\begin{tabular}{lccccc}
\hline & & \multicolumn{2}{c}{ Chassis } & \multicolumn{2}{c}{$\begin{array}{c}\text { Chassis installed } \\
\text { TAM }\end{array}$} \\
& & $V_{2}(\mathrm{mV})$ & $V_{3}(\mathrm{mV})$ & $V_{2}^{\prime}(\mathrm{mV})$ & $V_{3}^{\prime}(\mathrm{mV})$ \\
\hline 1 & 0 & 445 & 381 & 432 & 369 \\
2 & 5 & 845 & 365 & 522 & 331 \\
3 & 10 & - & - & 634 & 325 \\
4 & 15 & - & - & 795 & 309 \\
\hline
\end{tabular}

Note. $V_{2}, V_{2}^{\prime}$ are the plantar pressures of leg 2 , and $V_{3}, V_{3}^{\prime}$ are the plantar pressures of leg 3.

chassis installed an adjustable counterweight, making the barycenter of the whole chassis system changeable so that the chassis can achieve a higher stability and climb the steeper slope. The scheme of the flexible structure is also adopted by other chassis, such as the schemes proposed by Paul A and Mosterman [27]. Differing from the wheeled type and largescale equipment mentioned above, our manufactured prototype is small and lightweight, providing a role in limited working conditions under the forest. Hence, the legged structure is suitable for flexible operation and only four landing points are needed. Our chassis design with BAM has better adjustment performance, has low operator difficulty, and is more straightforward for manufacturing.

Also, a high-precision bearing is used in the BAM, which helps the counterweight rotate smoothly; and two sets of high-precision microguide and slide are adopted to achieve 
lower friction translation. The structure design reduces assembly difficulty, so that the appropriate counterweight can be replaced according to the slope inclination.

For BAM's barycenter curves, it is clear that the barycenter changes under the combined effect of three parameters. Rotation should be based on the translation. If the counterweight is at the center, rotation cannot change the barycenter position. While translation and vertical motion can change the barycenter, respectively, especially the vertical motion, it can effectively lower the height of the chassis barycenter even without translation and rotation. The counterweight can be reduced by up to $180 \mathrm{~mm}$. According to stability NESM [28], the barycenter's lower height could be viewed as better stability. The barycenter stays in fixed point and near the geometric center is conducive to have smaller shaking and vertical fluctuation [29]. The more times and distance the barycenter adjust, the greater the chassis vibration and consumption. That is the reason why we adopt the intermittent static gait to disperse the vibration of the chassis caused by barycenter adjustment. In addition, the barycenter should adjust according to the terrain, and the distance of barycenter adjustment should be reduced on the premise of keeping the chassis stable. When the slope is gentle, minor adjustment is suitable. While on the steep slope, we choose the maximum stability margin of barycenter adjustment to improve the motion stability.

Overall, it shows that the chassis with BAM is better than the standard chassis, especially for the barycenter position. As leg 4 is lifted, the former rolls over on a slope steeper than 5 degrees. The latter can be stable on a slope of 15 degrees, which is mainly affected by counterweight through changing the barycenter position. In theory, the chassis can keep stable on a steeper slope with a heavier counterweight. However, the heavier the counterweight is, the more torque the motor needs. Moreover, the friction between the foot end and the ground is also the factor affecting chassis stability. Thus, the heavier counterweight $s$ is not carried out in this paper.

\section{Conclusion}

In this paper, a forestry chassis with barycenter adjustable mechanism is proposed. According to the stability criterion of quadruped chassis, a control method is proposed to adjust the chassis barycenter by changing the parameters of counterweight to improve the chassis stability moving on the slope. The relationship between the range of counterweight displacement parameters $\left(R, \theta_{a}\right.$, and $\left.\sigma\right)$ and the barycenter position of the chassis is determined by mathematical analysis. The adjustment strategy is simulated in Adams, which realizes the barycenter adjustment. Finally, the test results show that the barycenter adjustable mechanism can adjust the chassis barycenter, and the motion stability of the chassis on a slope is improved. Through the test results analysis, the optimal adjustment of parameters is determined.

\section{Data Availability}

The data used to support the findings of this study are available from the corresponding author upon request.

\section{Conflicts of Interest}

The authors declare no conflicts of interest.

\section{Acknowledgments}

This research was funded by the National Key Research and Development Program (2018YFC0507102-02).

\section{References}

[1] Z. G. Wei and J. H. Liu, "Design of the chassis for a wheeled forest combined harvester," Journal of Guangxi University (Natural Science Edition), vol. 35, no. 2, pp. 263-268, 2010.

[2] Y. Wang, S. Z. Chen, and H. T. Li, "Design and experiment of high performance profiling terrain chassis with power train," Transactions of the Chinese Society of Agricultural Engineering, vol. 28, no. Suppl 1, pp. 39-44, 2012.

[3] J. Chen, J. F. Shi, Y. W. Li et al., "Present status and developing strategy of agricultural mechanization in Southwest China," Transactions of the Chinese Society of Agricultural Engineering, vol. 19, no. 5, pp. 1-5, 2003.

[4] X. Potau, M. Comellas, M. Nogués, and J. Roca, "Comparison of different bogie configurations for a vehicle operating in rough terrain," Journal of Terramechanics, vol. 48, no. 1, pp. 75-84, 2011.

[5] L. Zhou, L. Li, and X. F. Li, "Design and optimization of a multi-degree of freedom articulated mechanism," Modern Manufacturing Engineering, vol. 111, no. 9, pp. 120-123, 2009.

[6] Z. Sun, J. Liu, C. Yu, and J. Kan, "Stability analysis and gait planning for luffing wheel-legged robot during intelligent obstacle-surmounting process," Transactions of the Chinese Society of Agricultural Engineering, vol. 31, no. 16, pp. 1-7, 2015.

[7] Q. M. Gao, F. Gao, and L. Tian, "Design and development of a variable ground clearance variable wheel track self-leveling hillside vehicle power chassis ( V2-HVPC)," Journal of Terramechanics, vol. 56, pp. 78-90, 2014.

[8] Q. M. Gao, F. Gao, W. H. Zhou et al., "Stability analysis of hilly power platform with balance rocker suspension," Transactions of the CSAM, vol. 44, no. 2, pp. 291-293, 2013.

[9] W. Y. Zhang and L. Zhang, "Quadruped robot omni-directional walking on a slope based on static balance," in Proceedings of International Conference on Manufacturing and Automation, pp. 1436-1440, IEEE, Piscataway, NJ, USA, August 2012.

[10] L.-p. Wang and J.-z. Wang, "Foot trajectory generation and gait control method of a quadruped robot on uneven terrain based on zero moment point theory," Transaction of Beijing Institute of Technology, vol. 35, no. 6, pp. 601-606, 2015.

[11] J. H. Lee and J. H. Park, "Control for quadruped robots in trotting on horizontal and slanted surfaces," in Proceedings of the IEEE 9th Asian Control Conference (ASCC), pp. 1-6, Istanbul, Turkey, June 2013.

[12] X. Zeng, S. Zhang, H. Zhang, X. Li, H. Zhou, and Y. Fu, "Leg trajectory planning for quadruped robots with high-speed trot gait," Applied Sciences, vol. 9, no. 7, p. 1508, 2019.

[13] S. Seok, A. Wang, Y. C. Meng et al., "Design principles for highly efficient quadrupeds and implementation on the MIT Cheetah robot," in Proceedings of IEEE International Conference on Robotics \& Automation, pp. 3307-3312, Kalsruhe, Germany, May 2013. 
[14] C. Walker Jr, C. J. Vierck, and L. A. Ritz, "Balance in the cat: role of the tail and effects of sacrocaudal transaction," Behavioural Brain Research, vol. 91, no. 1-2, pp. 41-47, 1998.

[15] M. R. Alexander, "Biomechanics: leaping lizards and dinosaurs," Nature, vol. 481, no. 7380, 2012.

[16] T. Libby, T. Y. Moore, E. Chang-Siu et al., "Tail-assisted pitch control in lizards, robots and dinosaurs," Nature, vol. 481, no. 7380, p. 181, 2012.

[17] E. Chang-Siu, T. Libby, M. Tomizuka et al., "A lizard-inspired active tail enables rapid maneuvers and dynamic stabilization in a terrestrial robot," in Proceedings of IEEE RSJ International Conference on Intelligent Robots \& Systems, pp. 25-30, IEEE, San Francisco, CF, USA, September 2011.

[18] K. Graichen, S. Hentzelt, A. Hildebrandt, N. Kärcher, N. Gaißert, and E. Knubben, "Control design for a bionic kangaroo," Control Engineering Practice, vol. 42, no. sep, pp. 106-117, 2015.

[19] G. K. Hyun, G. L. Dong, and W. S. Tae, "Rolling stability enhancement via balancing tail for a water-running robot," Journal of Bionic Engineering, vol. 12, no. 3, pp. 395-405, 2015.

[20] J. Tao, h. Zang, and y. Cai, "Mechanism design and functional simulation of a new bionic cheetah robot," Machinery Design \& Manufacture, no. 4, pp. 214-216, 2015.

[21] D. H. Chadwick, A Beast the Color of Winter, Sierra Club Books, San Francisco, CF, USA, 1983.

[22] B. L. Smith, Life on the Rocks: A Portrait of the American Mountain Goat, University Press of Colorado, Boulder, CO, USA, 2014.

[23] T. Hobbs, "Travel in alpine terrain: energy expenditures for locomotion by mountain goats and bighorn sheep," Canadian Journal of Zoology, vol. 67, no. 10, pp. 2368-2375, 1989.

[24] R. T. Lewinson and D. J. Stefanyshyn, "A descriptive analysis of the climbing mechanics of a mountain goat (Oreamnos americanus)," Zoology, vol. 119, no. 6, pp. 541-546, 2016.

[25] F. Zhang, W. Wang, G. Y. Zhang et al., "Gait analysis of goat at different slopes and study on biomimetic walking mechanism," International Journal of Agricultural and Biological Engineering, vol. 9, no. 3, pp. 40-47, 2016.

[26] F. Zhang, W. Wang, J. Tong et al., "Moisture absorption properties of biomimetic laminated boards made from crosslinking starch/maize stalk fiber," International Journal of Agricultural and Biological Engineering, vol. 8, no. 2, pp. 65-71, 2015.

[27] F. J. Mosterman, Vehicle Comprising Jib and Counterweight Formed by Wheel and Motor, European Patent, 2012.

[28] S. Hirose, H. Tsukagoshi, and K. Yoneda, "Normalized energy stability margin and its contour of walking vehicles on rough terrain," in Proceedings of IEEE International Conference on Robotics \& Automation, IEEE, Seoul, Korea, May 2001.

[29] S. Zeng, Y. Tan, Z. Li et al., "Effect of mass-center position of spinal segment on dynamic performances of quadruped bounding with a flexible-articulated spine," Applied Sciences, vol. 10, no. 4, p. 1491, 2020. 